\title{
First Data From the Earth Radiation Budget Experiment (ERBE)
}

ERBE Science Team ${ }^{1}$

\begin{abstract}
The first data obtained from the Earth Radiation Budget Experiment (ERBE) are presented. These data include emitted infrared radiation, albedo, and estimated scene types for 15 November 1984 , as well as measurements of the 'solar constant'. Images from the GOES on the same day are included for comparison with the ERBE scene identification. On an instantaneous basis, clouds appear colder and more reflective than seems to have been noted before. The experiment data will be applied to several key studies of cloud-radiationclimate interactions.
\end{abstract}

\section{Introduction}

On 5 October 1984 the first of three satellites of the Earth Radiation Budget Experiment (ERBE) was launched by Space Shuttle Challenger. This satellite, the Earth Radiation Budget Satellite (ERBS), also carries the Stratospheric Aerosol and Gas Experiment II (SAGE-II). The second satellite carrying ERBE instruments, $N O A A-F$ (now $N O A A-9$ ), was launched on 12 December 1984. The third, NOAA-G, is expected to be launched in early to mid 1986 . Earth-viewing data collection from the $E R B S$ commenced with the opening of contamination covers in early November of 1984 . Thereafter, data have been obtained on a continuous basis including numerous solar calibrations and a period of along-track scanning for validating the directional models used in obtaining top-of-the-atmosphere fluxes from radiance observations. A similar schedule of data collection has been maintained on the NOAA-9 satellite instruments.

Even though the work of data collection and reduction has just begun, the ERBE data are already providing interesting new insights into the interaction between radiation and climate. For example, the instantaneous radiances observed

\footnotetext{
${ }^{1}$ ERBE Science Team principal investigators: B. R. Barkstrom, ERBE Experiment Scientist and Science Team Leader; Atmospheric Sciences Division, NASA Langley Research Center; A. Berroir, LMD, Paris; R. D. Cess, State University of New York at Stony Brook; A. Gruber, NOAA NESDIS; D. L. Hartmann, University of Washington, Seattle; F. B. House, Drexel University; E. F. Harrison, NASA Langley Research Center; F. O. Huck, NASA Langley Research Center; G. E. Hunt, Imperial College of Science and Technology, London; R. Kandel, CNRS, Paris; M. D. King, ERBE Project Scientist, NASA Goddard Space Flight Center; A. T. Mecherikunnel, NASA Goddard Space Flight Center; A. J. Miller, NOAA NMC; V. Ramanathan and J. Coakley, NCAR; E. Raschke, University of Cologne; G. L. Smith, NASA Langley Research Center; W. L. Smith, University of Wisconsin, Madison; and T. H. Vonder Haar, Colorado State University.

The complete Science Team is listed in the article by Barkstrom entitled "The Earth Radiation Budget Experiment (ERBE)."(January 1984 BULLETIN, 1170-1185.
}

with the ERBE scanner suggest that some storms have cloud albedos greater than 80 percent, while their infrared emission is close to that of blackbodies at stratospheric temperatures. Although such extremes in albedo and infrared emission have been expected on theoretical grounds, it has been difficult to find observational confirmation of these expectations. The instantaneous radiances from ERBE are also likely to prove useful in resolving which approximations are appropriate in the infrared-radiative-transfer models used in general circulation models. Indeed, initial examination of the data strongly suggests that the ERBE will provide some of the best calibrated broadband measurements of instantaneous radiances from the earth yet obtained.

Simultaneous observations by at least two satellites should considerably improve our knowledge of the diurnal variability of the earth's radiation fields. This knowledge is expected to be helpful in studies of the mechanisms of heat transfer within the atmosphere and oceans, as well as in estimating the monthly averages of reflected and emitted radiation. Thus, the ERBE data will add to our long-term record (which began in the 1950s) of energy exchange between earth and space (Suomi, 1958; Vonder Haar and Suomi, 1971; Raschke et al., 1973).

\section{Experiment description}

Each of the satellites on which ERBE is orbiting contains a nearly identical pair of instrument packages: a scanner and a nonscanner.

A nonscanner package contains five detectors: four viewing the earth and one observing the sun. Two of the earthviewing detectors observe from limb to limb; two observe a footprint about $1000 \mathrm{~km}$ in diameter at the top of the atmosphere. For each pair of nonscanner channels, there is one shortwave channel and one spectrally flat channel that is uniformly sensitive to both sunlight and terrestrial infrared radiation. The solar-constant observations are made with absolute radiometers nearly identical in design to those carried on the Solar Maximum Mission (Willson, 1979).

A scanner package has three telescopes: one for reflected sunlight, one for infrared energy emitted by the earth and its atmosphere, and one for sensing both wavelength bands together. These three telescopes rotate about a single axis, usually observing perpendicular to the satellite ground track. Their footprint at nadir is about $35 \mathrm{~km}$.

All of the instrument channels are designed to be traceable to both the solar constant and to the International Practical Temperature Scale of 1968 (IPTS-68). During the ground calibration of these instruments, the IPTS-68 traceability was obtained through platinum resistance thermometers embedded in a blackbody with a measured emissivity. An inte- 


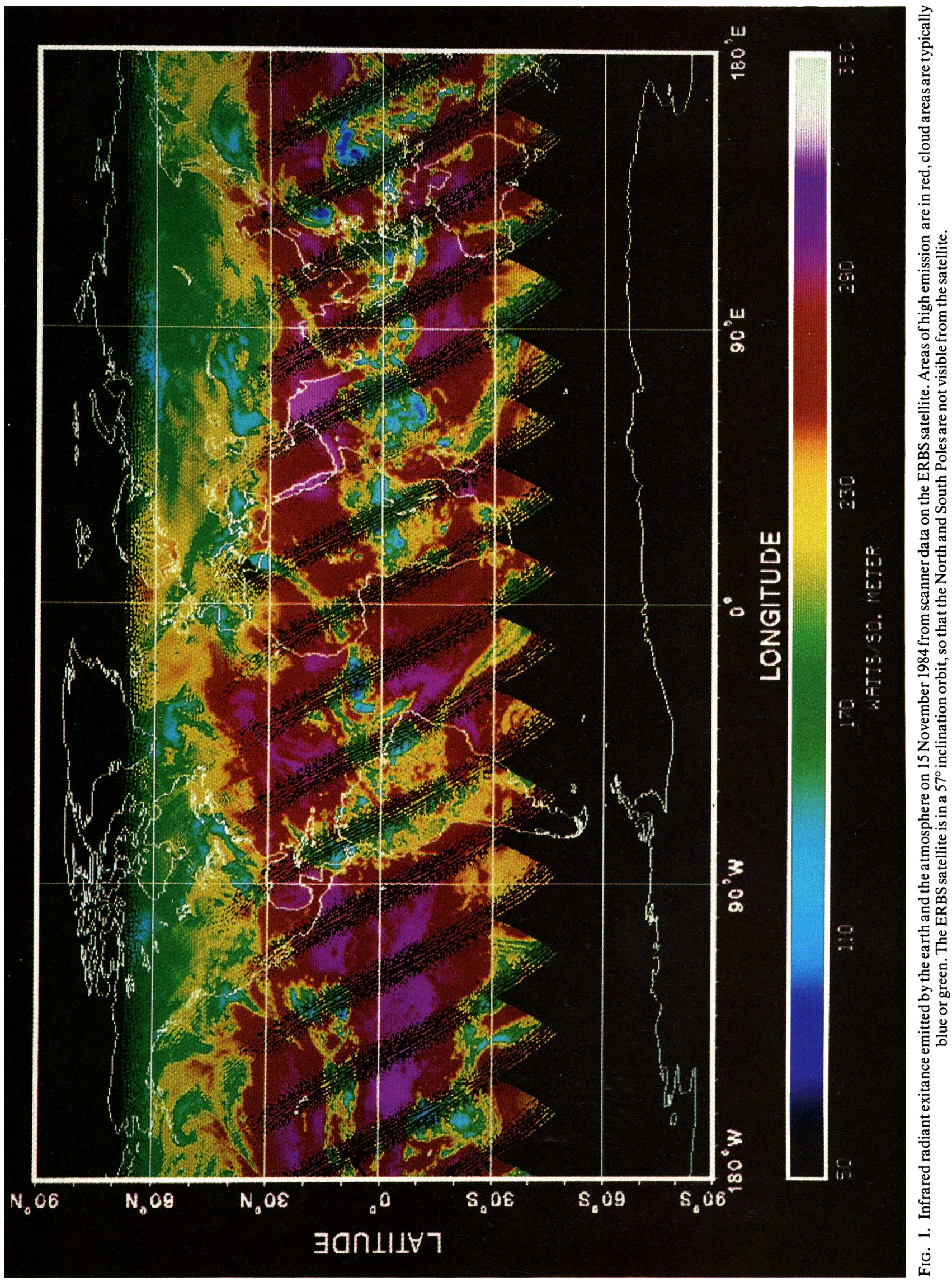


grating sphere served as a secondary transfer standard for the calibration of the shortwave channels. During flight, calibration is maintained through observations of internal blackbodies and of the Sun. A relative check of calibration is also provided by independently monitored tungsten lamps.

The data from these instruments are collected by NASA's Goddard Space Flight Center and by NOAA's National Environmental Satellite, Data, and Information Service. They are then transmitted to NASA's Langley Research Center, where the data-processing system converts them into calibrated radiances (for the scanner) and irradiances at the satellite (for the nonscanner). Thereafter, the data are reduced to instantaneous fluxes at the top of the atmosphere, and finally averaged over a month. The final data products will be made available to the scientific community after initial data validation and use by the ERBE Science Team. A considerably more-detailed overview of the experiment is provided by Barkstrom (1984), as well as by a series of articles by members of the ERBE Science Team in the Reviews of Geophysics (Hunt et al., 1986; House et al., 1986; Barkstrom and Smith, 1986; G. Smith et al., 1986; Kopia, 1986; Luther, et al., 1986; Brooks et al., 1986; Hartmann et al., 1986).

\section{Initial results from ERBE}

\section{a. Solar irradiance observations}

The first observations of the sun by the $E R B S$ instruments were made on 25 October 1984. For solar observations, the instrument is rotated in azimuth so that the sun moves through the field of view of the solar monitor. During this time, the earth-viewing channels are rotated away from the earth so that their optical axes point in the same direction as the solar monitor. The sun's light then shines on the detectors through solar ports on the side of the instrument.

The solar monitor uses a shutter to provide a reference source, while the earth-viewing channels observe space before and after the sun moves through the fields of view. The gain of these detectors is simply the reciprocal of the product of primary aperture area, resistance of the heater windings of the active cavity, cavity absorptivity, and the transmittance of the filter domes (on the shortwave channels). These quantities were measured on the ground. In addition, the ground calibration of the instruments provides an independently derived gain that compares with the "absolute" constants to within the uncertainties of the parameter measurements.

The solar constant obtained using the measured areas and resistances of the $E R B S$ solar monitor is approximately 1365 $\mathrm{W} \cdot \mathrm{m}^{-2}$ for the solar observations performed on 25 and 26 October. This value received further confirmation with observations obtained during the first solar calibration of the NOAA-9 measurements. Later solar observations have produced the same value with a standard deviation less than 1.5 $\mathrm{W} \cdot \mathrm{m}^{-2}$. This appears to represent a new level of agreement among independently constructed detectors. The value of the solar constant obtained by the ERBE detectors is within 0.3 percent of that obtained by other measurements from the Nimbus-7 (Hickey et al., 1980) and the Solar Maximum Mission (Willson, 1984) radiometers, and quite close to the value obtained from Spacelab Imeasurements (Crommelynck and Domingo, 1984).

\section{b. Instantaneous fluxes (exitances) from the top of the atmosphere}

Figure 1 shows the broadband, infrared flux (exitance) emerging from the top of the atmosphere at night during $15 \mathrm{No}$ vember 1984. Particularly interesting are high values observed off the coast of the Arabian peninsula and other clear ocean areas. The top of the atmosphere exitances from these regions are over $300 \mathrm{~W} \cdot \mathrm{m}^{-2}$.

These high values were also obtained on 9 November 1984 , and were high enough to catch the attention of the ERBE Science Team. Correlative meteorological sounding data obtained from the National Meteorological Center suggest that over the Gulf of Mexico on 9 November the middle troposphere had a tongue of dry air, which allowed the water vapor near the surface to radiate more effectively to space than would be the case with a moist troposphere. It may be that such instantaneous data from the ERBE instruments will be of considerable help in resolving differences among different radiative-transfer computation schemes used in climate models.

15 November 1984 was meteorologically interesting because there were three active tropical storms on that date. In the western Pacific, hurricanes Bill and Clara were slightly east of the Philippines. In the lower Caribbean, tropicalstorm Klaus was approaching Central America. These storms are visible in Fig. 1. A more-detailed view of the infrared flux over the Western Pacific is shown in Fig. 2. Bill and Clara are clearly visible, radiating to space at less than 75 $\mathrm{W} \cdot \mathrm{m}^{-2}$. This rate of emission corresponds to stratospheric temperatures.

ERBE also observes reflected sunlight, so that the geographic and temporal distribution of absorbed sunlight can be derived. The albedo, which is the fraction of incident sunlight reflected to space, is of considerable interest. The geographic distribution of instantaneous albedo on 15 November is shown in Fig. 3. Particularly noteworthy are albedos greater than $\mathbf{0 . 8 0}$, observed over the Intertropical Convergence Zone near Indonesia, over the Amazon Basin, and over the tropical storms mentioned earlier.

In addition to the high-resolution maps provided by the scanner, ERBE also has lower-resolution data provided by the nonscanner. These data will extend and overlap the nearly continuous climatological record of earth-radiationbudget data which began in 1975 with the Earth Radiation Budget (ERB) measurements on Nimbus-6 (W. Smith et al., 1977) and continued on Nimbus-7 (Jacobowitz et al., 1984).

\section{c. ERBE scene identifications}

As part of the inversion process, which relates observations at satellite altitude to radiation fields at the top of the atmos-

FIG. 2. (facing page) Infrared radiant exitance observed by the ERBS scanner on 15 November 1984 at night over the western Pacific. Tropical hurricanes Bill and Clara are clearly visible in this image emitting very low amounts of radiation. The blue areas in the center of the storms are emitting less than $75 \mathrm{~W} \cdot \mathrm{m}^{-2}$, corresponding to stratospheric temperatures. 


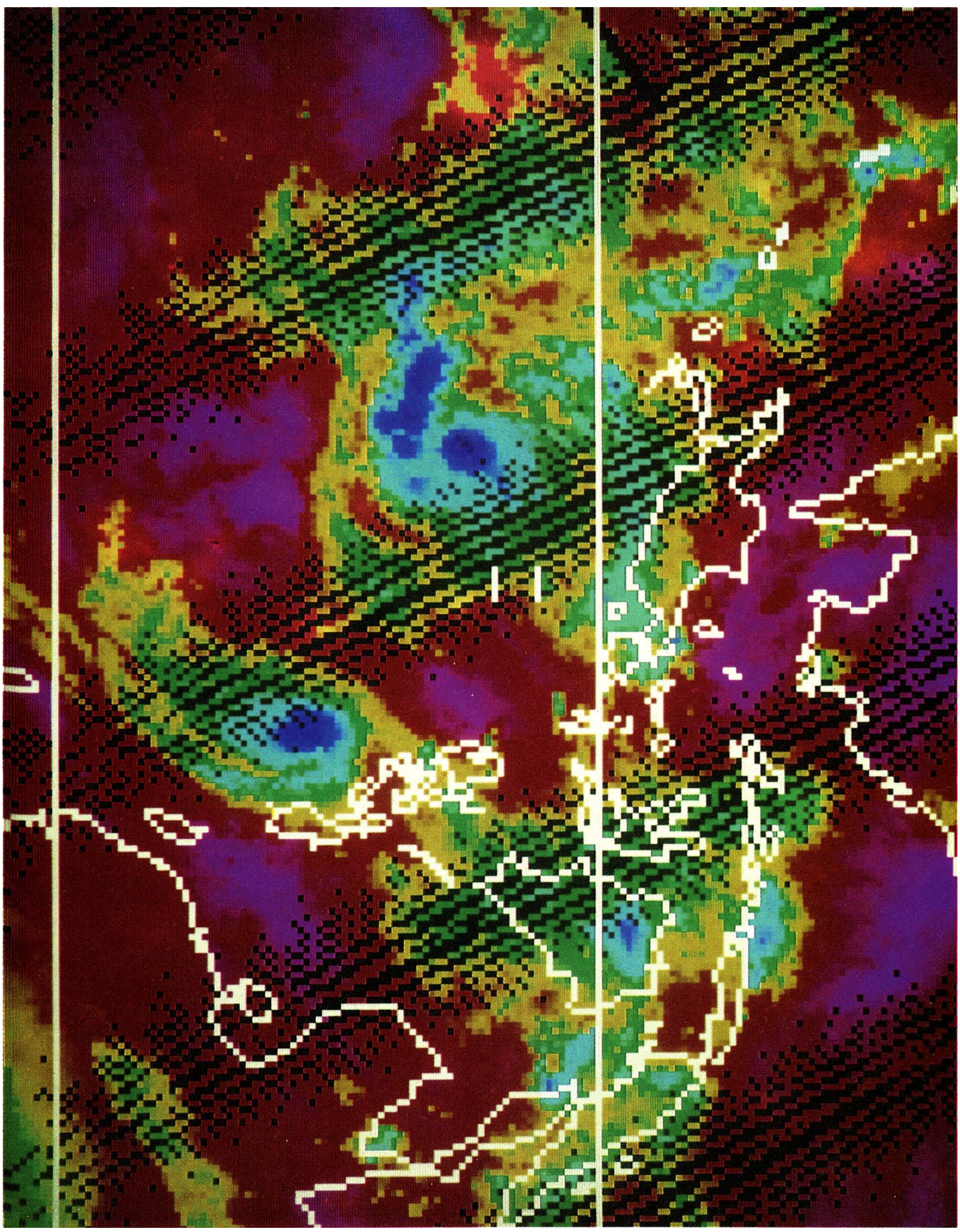




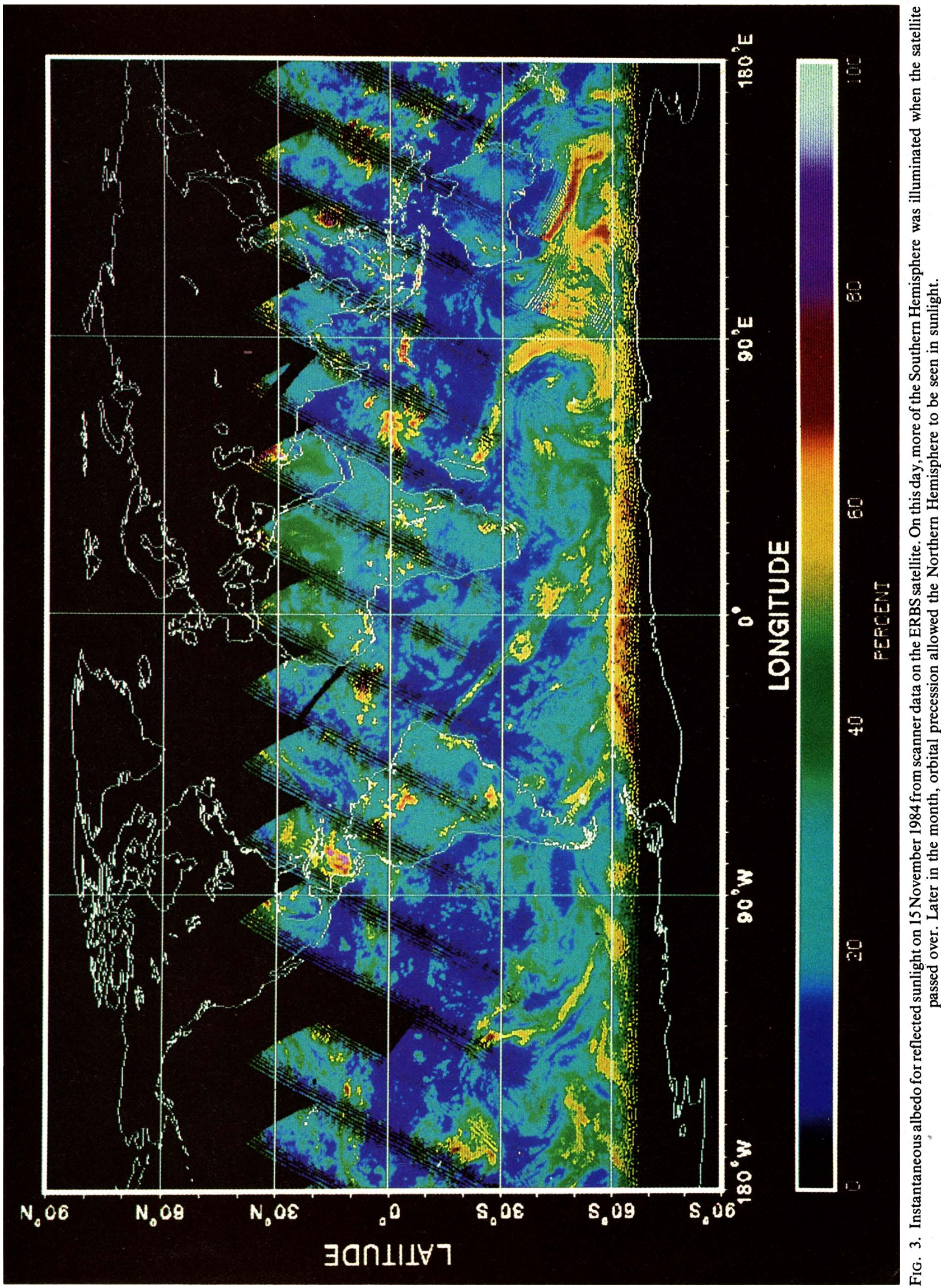



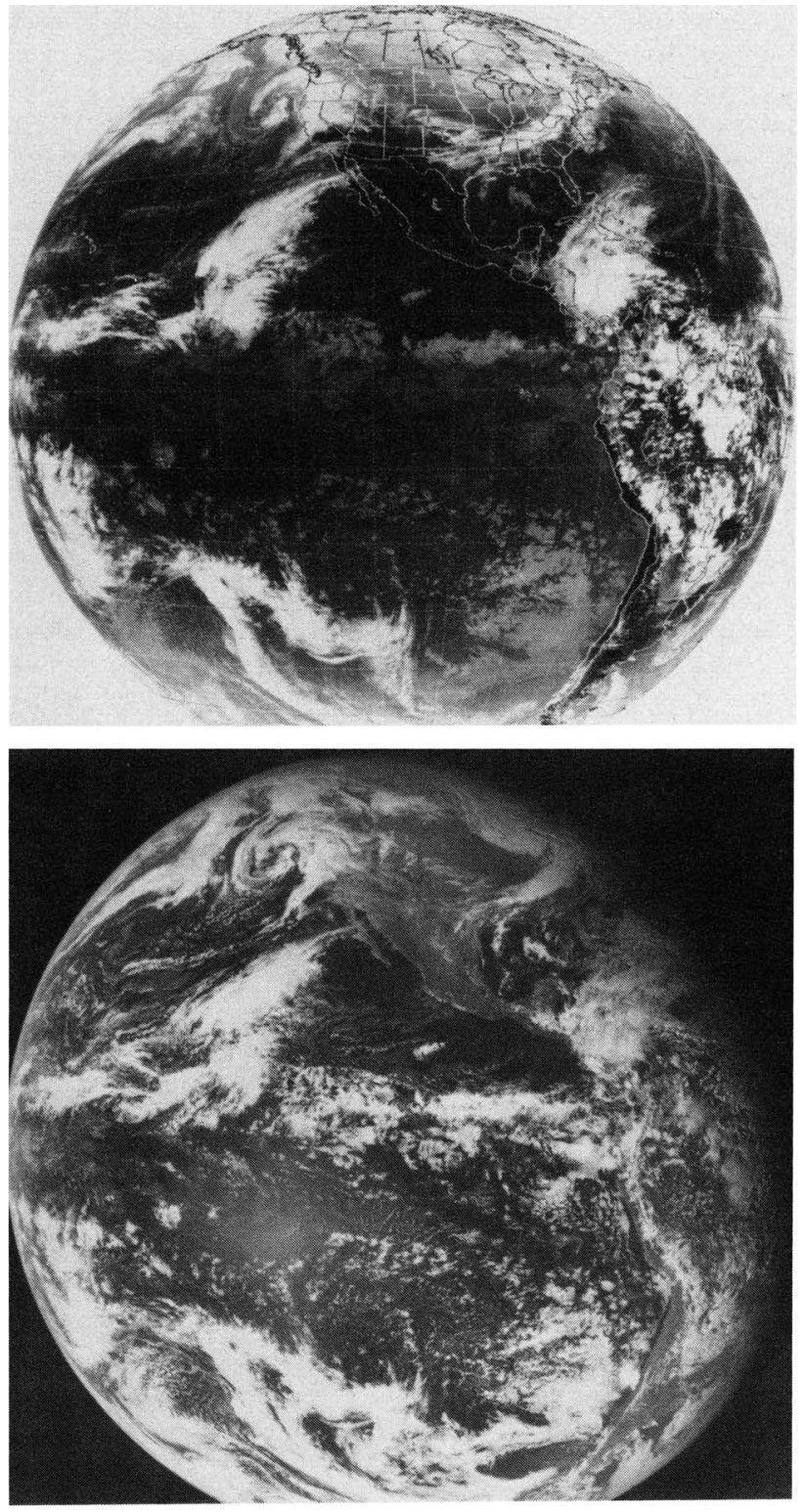

FIG. 4. (top) GOES Infrared Image for 2100 UT on 15 November 1984. Clouds appear as white; hot desert surfaces as dark. Note the general agreement between the clouds in this image and the ERBE scene type in the figure on the cover of this issue. (bottom) GOES Visible Image for 2100 UT on 15 November 1984. This image shows the earth more or less as the eye would see it: clouds are white, the ocean is dark. Sun glint from the ocean appears as a brightening slightly to the left of the center of this image. The solar illumination of tropical-storm Klaus corresponds with that in the ERBE scene identification shown in the cover figure.

phere, the earth is classified into five geographic scene types: ocean, land, desert, snow, and coast. For each geographic type the instantaneous observations are further classified into clear, partly cloudy, mostly cloudy, and overcast. The data for this classification and for the directional modeling come primarily from angular models developed from the Nimbus-7 ERB measurements (Taylor and Stowe, 1984).

It should be noted that the precise connection between the normal meteorological understanding of partially cloudy or overcast scenes and the ERBE scene types is not completely established. Similar caveats hold with respect to the relationship between the ERBE scene types and cloud identifications obtained from other meteorological satellite data. Indeed, one of the major tasks of ERBE validation will be to determine the precise nature of these correlations.

Despite the important caveats regarding the quantitative interpretation of the ERBE scene types, it is encouraging that they bear a prima facie resemblance to images obtained by the narrow-band channels on geostationary satellites. The figure on the cover of this issue shows a global image obtained from the ERBE scanner scene identification during 15 November 1984. Figure 4 (top) shows a GOES IR image and 4 (bottom) a GOES visible image for 2100 UT on this date. The ERBE data cover a broader range of time than does the single pair of GOES images, although each orbital pass covers only a portion of a GOES image during the period when they are coincident.

It is of considerable interest to note the marked similarities between the two types of images. In particular, the band of ERBE overcast scenes over central South America, northern North America, the eastern Pacific, and the equatorial $\mathrm{Pa}$ cific seem to agree well with the visual appearance of cold, bright regions that are normally associated with clouds. Indeed, tropical-storm Klaus stands out dramatically in the slanting light of late afternoon, corresponding to the illumination in the ERBS overpasses.

The resemblance between the ERBE scene identification and the geostationary images will be quantitatively examined as part of the ERBE validation. This work will involve not only the United States' GOES data, but data from METEO$S A T$ and from AVHRR as well. It is important to note that these auxiliary data sources contribute additional temporal sampling. The METEOSAT data are important because they include such important climate regions as the Sahara and the Sahel.

\section{Comments on the ERBE data}

Much work remains to be done before the top-of-the-atmosphere and monthly averaged data from ERBE are finally archived. Even at this time, however, the ERBE data appear to be a new, highly calibrated source of information regarding the earth's radiation fields. It may be hoped that the data collection will continue beyond the design life goal of two years for these instruments, so that the record of the earth's radiation budget may continue to be studied.

Acknowledgments. It is not possible in such a brief space to give credit to all of the individuals and organizations that have contributed to the success of ERBE. J. E. Cooper, the ERBE experiment manager, M. R. Luther, the ERBE nonscanner engineer, and L. P. Kopia, the ERBE scanner engineer, have made major contributions to the overall management and technical design of the instruments. The $E R B S$ spacecraft contractor at Ball Aerospace Systems Division and the Space Group at TRW contributed to the flawless operation of the satellite and the instruments. Ray Hesser, Cindy Hendricks, and Andy Castineiras at TRW contributed enormously to the knowledge required for understanding the instruments. The ground command and control centers at GSFC and at NOAA have also contrib- 
uted significantly. In addition, a major contribution to the success of this measurement program lies with the ERBE Data Management Team at NASA's Langley Research Center. This group, headed by James F. Kibler and assisted by personnel at OAO Corp., Kentron International, System Development Corporation Inc., and the Analysis and Computation Division of LaRC, have made it possible to produce the images that appear in this article.

Finally, the Science Team has a number of Co-Investigators without whose contributions the data would not appear. The Instrument Working Group (including Co-I's N. Halyo, S. Choi, and D. Chrisman) developed and implemented the instrument data reduction algorithms. The Inversion Working Group (particularly Co-I's L. Avis, R. N. Green, J. T. Suttles, B. A. Wielicki, R. Taylor, and L. Stowe) developed and implemented the inversion data reduction algorithms and data bases. The Averaging Working Group (particularly Co-I's D. Brooks and P. Minnis) developed and implemented the averaging algorithms and data-base software.

\section{References}

Barkstrom, B. R., 1984: The Earth Radiation Budget Experiment (ERBE). Bull. Amer. Meteor. Soc., 65, 1170-1185.

Barkstrom, B. R., and G. L. Smith, 1986: The Earth Radiation Budget Experiment: Science and implementation. Rev. Geophys. (in press.)

Brooks, D. R., E. F. Harrison, P. Minnis, and J. T. Suttles, 1986: Development of algorithms for understanding the temporal and spatial variability of the earth's radiation balance. Rev. Geophys. (in press.)

Crommelynck, D., and V. Domingo, 1984: Solar irradiance observations. Science, 225, 180.

Hartmann, D. L., V. Ramanathan, A. Berroir, and G. E. Hunt, 1986: Earth radiation budget science. Rev. Geophys. (in press.)

Hickey, J. R., L. L. Stowe, H. Jacobowitz, P. Pelligrino, R. H. Maschoff, F. B. House, and T. H. Vonder Haar, 1980: Initial solar irradiance determinations from Nimbus 7 cavity radiometer measurements. Science, 208, 281-283.
House, F. B., A. Gruber, G. E. Hunt, and A. T. Mecherikunnel, 1986: History of satellite missions and measurements of the earth radiation budget. Rev. Geophys. (in press.)

Hunt, G. E., R. Kandel, and A. T. Mecherikunnel, 1986: A history of pre-satellite investigations of the earth's radiation budget. Rev. Geophys. (in press.)

Jacobowitz, H., H. V. Soule, H. L. Kyle, F. B. House, and the NIMBUS 7 ERB Experiment Team, 1984: The Earth Radiation Budget (ERB) Experiment: An Overview. J. Geophys. Res., 89, 5021-5038.

Kopia, L. P., 1986: The Earth Radiation Budget Experiment scanner instrument. Rev. Geophys. (in press).

Luther, M. R., J. E. Cooper, and G. R. Taylor, 1986: The Earth Radiation Budget Experiment nonscanner instrument. Rev. Geophys. (in press.)

Raschke, E., T. H. Vonder Haar, W. R. Bandeen, and M. Pasternak, 1973: The annual radiation balance of the earth-atmosphere system during 1969-1970 from Nimbus 3 measurements. J. Atmos. Sci., 30, 341-364.

Smith, G. L., R. N. Green, E. Raschke, L. M. Avis, B. A. Wielicki, and R. Davies, 1986: Inversion methods for satellite studies of the earth's radiation budget: development of algorithms for the ERBE missions. Rev. Geophys. (in press.)

Smith, W. L., D. T. Hilleary, H. Jacobowitz, H. B. Howell, J. R. Hickey and A. J. Drummond, 1977: Nimbus-6 Earth Radiation Budget Experiment, Appl. Opt., 16, 306-318.

Suomi, V. E., 1958: The radiation balance of the Earth from a satellite. Ann. IGY, 1, 331-340.

Taylor, V. R. and L. L. Stowe, 1984: Reflectance characteristics of uniform earth and cloud surfaces derived from NIMBUS-7 ERB. J. Geophys. Res., 89, 4987-4996.

Vonder Haar, T. H. and V. E. Suomi, 1971: Measurements of the Earth's radiation budget from satellites during a five-year period. Part 1: Extended time and space means. J. Atmos. Sci., 28, 305-314. Willson, R. C., 1979: Active Cavity Radiometer Type IV. Appl. Opt., 18, 179-188.

Willson, R. C., 1984: Measurements of solar total irradiance and its variability. Space Sci. Rev., 38, 203-242. 